\title{
Improving vitamin D content in pork meat by UV bio-enrichment
}

\author{
H.R. Neill ${ }^{1}$, C.I.R. Gill ${ }^{1}$, E.J. McDonald ${ }^{2}$, W.C. McRoberts ${ }^{3}$, E.J. Rosbotham ${ }^{1}$, R. Boland ${ }^{1}$ and \\ L.K. Pourshahidi ${ }^{1}$ \\ ${ }^{1}$ Nutrition Innovation Centre for Food and Health (NICHE), School of Biomedical Sciences, Ulster University, \\ Coleraine, UK, \\ ${ }^{2}$ Devenish Nutrition Ltd., Belfast, UK and \\ ${ }^{3}$ Agri-Food and Biosciences Institute, Belfast, UK
}

Vitamin D deficiency is prevalent worldwide and the COVID-19 pandemic has highlighted the fundamental need to ensure optimal vitamin D status to help maintain immune function ${ }^{(1)}$. Given the marked variation in seasonally-induced cutaneous synthesis, habitually low dietary vitamin D intakes $(2-4 \mu \mathrm{g}$ /day) and the generally low uptake of supplementation at the population level, identification of alternative food-based strategies are urgently warranted. Bio-enrichment is a growing area of research, with particular interest in the use of fortified animal feed and/or UV exposure to naturally increase vitamin D content in meat ${ }^{(2)}$. Since meat contributes the highest percentage to total vitamin D intake ${ }^{(3)}$, enriched pork meat may offer a plausible vehicle for bio-enrichment. The aim of the current study was to determine the impact of daily UVB exposure to bio-enrich vitamin D in pork meat.

Two independent studies were conducted in Duroc pigs (Study $1 \mathrm{n}=48$; Study $2 \mathrm{n}=20$ ). In both studies, 10-week old pigs were raised indoors and exposed daily to narrowband UVB radiation $(440 \mu \mathrm{W} / \mathrm{cm} 2$, exposure time increasing from Study 1 to 2 , without erythema) for 11 weeks or control (no UVB exposure) until slaughter. All pigs were fed the maximum permitted 2000 IU vitamin $\mathrm{D} 3 / \mathrm{kg}$ feed. Serum samples were obtained at baseline, midpoint and endpoint and following slaughter, loin meat was cooked. All samples were analysed for vitamin D3, vitamin D2, 25(OH)D3 and 25(OH)D2 $(\mu \mathrm{g} / \mathrm{kg})$ via LC-MS. Total vitamin D activity was calculated as vitamin $\mathrm{D}+[25(\mathrm{OH}) \mathrm{D} \times 5]$.

In Study 1, UVB exposure significantly increased serum 25(OH)D3 concentrations in the pigs (mid and endpoint, $\mathrm{p}<0.05$ ) but did not alter total vitamin D activity, vitamin D3 nor $25(\mathrm{OH}) \mathrm{D} 3$ concentration in pork meat compared to control ( $\mathrm{p}>0.05$ ). In Study 2 , the higher UVB exposure time resulted in a significantly higher serum $25(\mathrm{OH}) \mathrm{D} 3$ concentration (mid and endpoint, $\mathrm{p}<0.05$ ) and in all measures of vitamin D3 in pork loin, compared to control. Specifically, median (IQR): vitamin D activity [22.88 (20.77-25.10) vs. $14.50(13.35-17.59) \mu \mathrm{g} / \mathrm{kg}, \mathrm{p}<0.001]$; vitamin D3 [11.97 (9.53-14.60) vs. 6.03 (5.58-6.65) $\mu \mathrm{g} / \mathrm{kg}, \mathrm{p}<0.001]$ and $25(\mathrm{OH}) \mathrm{D} 3 \mathrm{concentra-}$ tion $[2.09(2.02-2.49)$ vs. $1.65(1.45-2.19) \mu \mathrm{g} / \mathrm{kg}, \mathrm{p}=0.042]$. Pigs remained healthy during both studies and developed no signs of erythema.

Bio-enrichment by UVB radiation (Study 2 but not Study 1) provides an effective strategy to safely increase the naturally occurring vitamin D3 and 25(OH)D3 concentrations in pork loin. This effective enhancement strategy will inform new product development to produce value-added vitamin D bio-enriched products that may help consumers bridge the gap between vitamin D recommendations and current intakes, thus reducing rates of hypovitaminosis D. Further research is needed to identify the optimum UVB exposure duration and confirm the efficacy of vitamin D bio-enriched pork in human intervention studies.

\section{Acknowledgements}

This work was funded as part of a Department for the Economy (DfE) Co-operative Awards in Science and Technology (CAST) PhD studentship, supported by Devenish Nutrition Limited.

\section{References}

1. Lanham-New SA, Webb AR, Cashman KD, et al. (2020) BMJ Nutr, Prevention \& Health

2. Spiro A \& Buttriss JL (2014) Nutr Bull 39, 322-350

3. Public Health England (2018) NDNS results from years 7 and 8 (combined): data tables 